\title{
Tratamiento endourológico de la litiasis del tracto urinario superior en la infancia
}

\author{
Romero Otero J*, Gómez Fraile A, Feltes Ochoa JA*, Fernández I**, \\ López Vázquez F, Aransay Bramtot A
}

\begin{abstract}
Sección de Urología Pediátrica. *Servicio de Urología. Hospital Universitario 12 de Octubre. Madrid. **Servicio de Urología. Hospital Universitario de la Princesa. Madrid.
\end{abstract}

Actas Urol Esp. 2007;31(5):532-540

\section{RESUMEN}

TRATAMIENTO ENDOUROLÓGICO DE LA LITIASIS DEL TRACTO URINARIO SUPERIOR EN LA INFANCIA

Introducción: La litiasis en la infancia es una patología en ascenso. En nuestro medio hoy en día se caracteriza por ser de oxalato cálcico y ureteral. Así se ha convertido en un problema muy similar al de la edad adulta, por lo tanto de está haciendo un esfuerzo para aplicar la experiencia y eficacia existente en ese grupo en la edad pediátrica. La litotricia extracorpórea por ondas de choque es ya la primera opción terapéutica y el abordaje endourológico debe convertirse en un procedimiento habitual. Exponemos nuestra técnica y experiencia.

Material y métodos: Estudio retrospectivo y descriptivo de los niños diagnosticados de litiasis en el tracto urinario superior, tratados mediante endourologia en nuestro centro, desde enero de 1992 hasta enero del 2005. De su historial clínico hemos recogido las siguientes variables: $1^{\circ}$ Variables preoperatorias: Edad, sexo, manifestación clínica predominante al diagnóstico, tamaño de la litiasis en milímetros $(\mathrm{mm})$ y localización de la misma en el tracto urinario (dividimos éste en 1.) renal, 2.) tercio ureteral proximal, 3.) tercio ureteral medio y 4.) tercio ureteral distal). $2^{\circ}$ Variables operatorias: Tipo de intervención endourológica practicada, consideramos la vía de acceso: anterógrada o nefrolitotomía percutánea (NLP) y retrógrada o ureterorrenoscopia (URSC) y si se requirió o no la reconversión a cirugía a cielo abierto. $3^{\circ}$ Variables postoperatorias: tiempo de evolución, presencia de complicaciones precoces o tardías y el estado del paciente en la última revisión en consulta, donde valoramos: situación clínica y la ECO.

Resultados: En este periodo hemos realizado 7 procedimientos endourológicos en 6 pacientes, 4 niños y 2 niñas. La mediana de edad es de 4 años con un rango de 2,5-13 años. La manifestación clínica más frecuente fue el dolor lumboabdominal (4/7). La hematuria macroscópica y una infección del tracto urinario fueron las otras formas de presentación. El tamaño de los cálculos oscilaba entre 4 y 7 $\mathrm{mm}$, con la excepción de un cálculo coraliforme. La localización de la litiasis en la vía urinaria fue: ureteral distal 5 casos, ureteral proximal 1 caso y coraliforme el último.

Los procedimientos que hemos realizado son: una NLP para el tratamiento del cálculo coraliforme y 6 URSC. Con el acceso percutáneo se consiguió la extracción de toda la masa litiásica. En 3 de las 6 URSC se consiguió la extracción de la litiasis completa y no se necesitó de ningún tratamiento adicional. Esto representa una tasa de éxito con ambas técnicas del 57\% (5/7). En los 3 procedimientos restantes tuvimos que reconvertir a cirugia a cielo abierto para la extracción de la litiasis.

Con un tiempo de seguimiento que varia de 1 a 13 años todos los niños están asintomáticos, la ECO dentro de la normalidad y con buena función renal. No han presentado complicaciones ni a corto ni a largo plazo.

Conclusiones: Las técnicas endourológicas son factibles en la edad pediátrica. El desarrollo de material endourológico y la experiencia del cirujano son las claves para su desarrollo.

Palabras clave: Urolithiasis Litiasis urinaria. Endourologia. Litiasis pediátrica. Nefrolitotomía, Ureterorrenoscopia.

\section{ABSTRACT}

THE LITHIASIS IN THE UPPER URINARY TRACT IN CHILDREN: ENDOUROLOGICAL TREATMENT

Introduction: Urolithiasis in the pediatric age is a growing problem. In the developed world they are of calcium oxalate and in the upper urinary tract. It is very similar to the presentation of lithiasis in adults, so we have to make an effort to apply the experience in this age to the children. The shock wave lithotripsy is admitted as the first treatment for this pathology in the pediatric population already. The endourological approach must be use as a common approach in this group. We present our technique and experience.

Material and methods: A retrospective, descriptive study of the children diagnosed of lithiasis in the upper urinary tract that were treated by an endourological technique in our centre between January 1992 and January 2005. We gathered data on: 1.) Preoperative: age, sex, clinical manifestations, size $(\mathrm{mm})$ and position of the lithiasis (we divided the upper urinary tract in: renal, proximal third, medial third and distal third) 2.) Operative variable: endourological technique: percutaneus neprolithotomy or ureteroscopy. Reconversion to open surgery. 3.) Postoperative variables: time since surgery, complications and the current state of the patient (ultrasonography and renal function).

Results: Seven children, 4 boys and 2 girls with an age range of 2,5 to 14 years, underwent operation using an endourological technique. Lumboabdominal pain was the main clinical manifestation (4/7). The lithiasis size was 4-7mm, with the exception of a staghorn calculis. The calculis were: 5 ureteral proximal, 1 ureteral distal and one in the kidney (staghound stone).

We performed one percutaneus neprolithotomy for the staghorn calculi. We removed completly the stone and had no complications. The 6 other procedures were ureteroscopies. In 3 of them we removed the calculi ( $4 / 7$ success rate of $57 \%)$. The rest procedures we needed to transform in open surgery. With a following time of 1-13 years all of them are asymptomatic, and with ultrasonography and renal function in the normal limits. We did see no complications.

Conclusion: The endourological treatment for urolithiasis in pediatric patients is possible but must be individualized in each case. With the development of new endourological material and more surgical experience this technique will be to the reach of the most of the urologists.

Keywords: Urolithiasis. Urinary lithiasis. Endourology. Lithiasis in children. Percutaneus nefrolithotomy. Ureteroscopy. 
$\mathrm{E}$ n la edad adulta la incidencia de la urolitiasis en el mundo occidental es alta $(0-5 \%)^{1}$. Se trata de una patología recidivante en multitud de casos. Esto ha provocado que se haya acumulado una gran experiencia y eficacia en el manejo de esta enfermedad. Para su tratamiento se emplean procedimientos poco invasivos, altamente resolutivos y muy seguros.

La litiasis del tracto urinario en la edad pediátrica en nuestro medio está aumentando su frecuencia $^{1}$. En los países desarrollados ha cambiado su epidemiología y ha pasado de ser de urato y vesical, a ser de oxalato cálcico y ureteral ${ }^{2}$. Por lo tanto se ha convertido en una patología muy similar a la del adulto. Esto está provocando un esfuerzo por trasladar el abordaje terapéutico de este grupo de edad al niño. Se quieren conseguir altas tasas de curación con abordajes minimamente invasivos y sin dejar secuelas. La litotricia extracorpórea por ondas de choque (LEOC) es ya el procedimiento de elección para tratar esta enfermedad en ambas edades. Tras su fracaso disponemos de múltiples opciones terapéuticas. Las técnicas endourológicas son las menos invasivas y al igual que en el adulto deben convertirse en el paso siguiente. La poca homogeneidad en edad y talla existente entre los prepúberes, junto con la desproporción en tamaño entre el material endourológico y el niño hace que todavía hoy no se emplee este abordaje rutinariamente. Exponemos nuestra técnica y experiencia inicial para este procedimiento.

\section{MATERIAL Y MÉTODOS}

Realizamos un estudio retrospectivo y descriptivo de los individuos en edad pediátrica, diagnosticados de litiasis en el tracto urinario superior, que han sido tratados mediante una técnica endourológica en nuestro centro, en el intervalo de tiempo comprendido desde enero de 1992 hasta enero del 2005 (13 años). De su historial clínico hemos recogido las siguientes variables:

1. Variables preoperatorias: Edad, sexo, manifestación clínica predominante al diagnóstico, tamaño de la litiasis en milímetros $(\mathrm{mm})$ y localización de la misma en el tracto urinario (dividimos éste en: 1) Renal, 2) Tercio ureteral proximal, 3) Tercio ureteral medio y 4) Tercio ure- teral distal). Para determinar estas últimas características hemos utilizado los datos que se obtuvieron en las pruebas de imagen que realizamos para el diagnóstico; a todos los enfermos les realizamos una ecografía del aparato urinario (ECO) y una radiografia simple de abdomen (Rx), en casos seleccionados complementamos el estudio con una urografía intravenosa (u.i.v) y/o tomografía axial computerizada (TAC).

2. Variables operatorias: Tipo de intervención endourológica practicada, consideramos la vía de acceso: anterógrada o nefrolitotomía percutánea (NLP) y retrógrada o ureterorrenoscopia (URSC) y si se requirió o no la reconversión a cirugía a cielo abierto.

3. Variables postoperatorias: tiempo de evolución, presencia de complicaciones precoces o tardías y el estado del paciente en la última revisión en consulta, donde valoramos: situación clínica y la ECO.

Todos los enfermos fueron sometidos previamente a tratamiento con LEOC y ante su fracaso se optó por la cirugía endourológica.

Las técnicas que hemos empleado se pueden dividir en dos grupos en función de la vía de acceso: NLP o anterógrada y URSC o retrógrada.

1. Para el acceso percutáneo posicionamos al niño en primer lugar en decúbito supino, realizamos una cistoscopia (cistoscopio de 10,5 Fr., que dispone de un canal recto de trabajo), introducimos un catéter ureteral (variable en función de la talla del niño) y procedemos a introducir contraste para opacificar la vía urinaria (así disponemos de un "dibujo" de esta y nos ayuda a la localización exacta del cálculo), posteriormente fijamos el catéter a una sonda vesical tipo Foley (el tamaño también varía en función de la talla del niño) y para finalizar posicionamos al enfermo en decúbito prono. A continuación con la vía rellena de contraste y opcionalmente de azul de metileno, bajo control fluoroscópico, puncionamos el cáliz inferior y ayudándonos de dilatadores telescópicos tipo Alken) (Fig. 1) ampliamos el trayecto. Colocamos una guía de seguridad (así siempre tenemos tutorizado el acceso) y emplazamos un amplatz que facilita el paso de todo tipo de instrumental (Fig. 2). Introducimos el nefroscopio y 


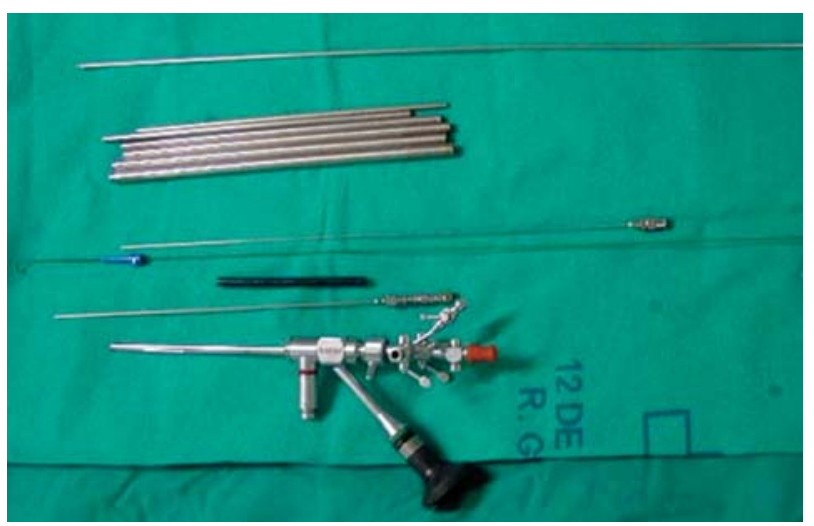

FIGURA 1: Material para realización de nefrolitotomía percutánea. Se visualiza nefroscopio y dilatadores de "Alken".

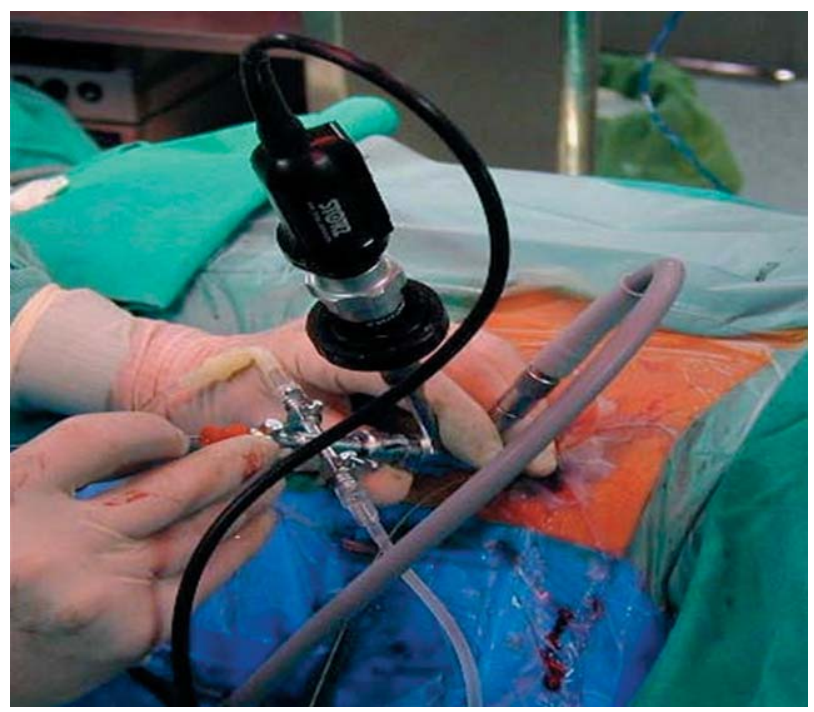

FIGURA 2: Nefroscopio introducido a través de un amplatz para acceder a la vía urinaria.

para la fragmentación y extracción del cálculo nos ayudamos de: pinzas de cuerpo extraño, cesta de dormia y de Lithoclast ${ }^{\circledR}$. Reemplazamos el catéter ureteral por un catéter doble $\mathrm{J}$ y además ayudamos al drenaje de la pelvis renal mediante el emplazamiento de una nefrostomía por el trayecto percutáneo. El doble $\mathrm{J}$ lo retiramos a las 6 semanas de la intervención y la nefrostomía antes del alta.

2. Para la URSC, colocamos al paciente en decúbito supino, realizamos una cistoscopia (cistoscopio de 10,5 Fr) y así cateterizamos el meato ureteral del riñón afecto con una guía hidrofilica, sobre ella pasamos un catéter balón de angioplastia de $5 \mathrm{Fr}$, con una longitud de $4 \mathrm{~cm}$ y un diámetro máximo de $5 \mathrm{~mm}$ que se hincha para dilatar el meato. Empleamos cistoscopio de 9,5 Fr a modo de ureterorrenoscopio, y así a través de su canal de trabajo podemos hacer pasar, al igual que en el acceso percutáneo, diferentes instrumentos para fragmentar y extraer la litiasis. Al finalizar dejamos emplazado un catéter tipo doble $\mathrm{J}$ número 4 durante 6 semanas.

\section{RESULTADOS}

En el periodo de tiempo comprendido entre Enero de 1992 y Enero 2005 hemos realizado 7 procedimientos endourológicos en 6 pacientes, 4 niños y 2 niñas. La mediana de edad es de 4 años con un rango de 2,5-13 años (Tabla 1). La manifestación clínica más frecuente fue el dolor lumboabdominal que lo presentaron 4 de ellos (57\%). La hematuria macroscópica y una infección del tracto urinario fueron las otras formas de presentación.

En todos los enfermos se emplearon la ecografía y la radiografía simple de abdomen como pruebas diagnósticas de imagen, así constatamos que el tamaño de los cálculos oscilaba entre 4 y $7 \mathrm{~mm}$, con la excepción de un cálculo coraliforme. La localización de la litiasis en la vía urinaria en el momento de la intervención fue: ureteral distal 5 casos, ureteral proximal 1 caso y coraliforme el último.

Los procedimientos que hemos realizado son: una NLP para el tratamiento del cálculo coraliforme y 6 URSC. Con el acceso percutáneo se consiguió la extracción de toda la masa litiásica y no se requirió la realización de LEOC posterior. En 3 de las 6 URSC se consiguió la extracción de la litiasis completa y no se necesitó de ningún tratamiento adicional. Esto representa una tasa de éxito con ambas técnicas del 57\% (4/7). En los 3 procedimientos restantes tuvimos que reconvertir a cirugía a cielo abierto para la extracción de la litiasis. Uno de los casos se trataba de una litiasis de $4 \mathrm{~mm}$ en uréter proximal, y con el instrumental del que disponemos fue imposible ascender lo suficiente para manipular el cálculo. En los otros 2 casos la litiasis era de gran tamaño, ambas mayores de $4 \mathrm{~mm}$ y no se consiguió completar la fragmentación y extracción de la misma, por lo que se decidió la reconversión (previamente el tratamiento con LEOC había fracasado). 
Tabla 1.

Resumen de las características de los enfermos.

\begin{tabular}{|c|c|c|c|c|c|c|c|}
\hline Sexo & $\begin{array}{l}\text { Edad } \\
\text { (años) }\end{array}$ & Clinica & $\begin{array}{c}\text { Tamaño } \\
\text { Litiasis }(\mathbf{m m})\end{array}$ & $\begin{array}{c}\text { Localización } \\
\text { litiasis }\end{array}$ & Procedimiento & Reconversión & $\begin{array}{r}\text { Estado } \\
\text { actual }\end{array}$ \\
\hline Varón & 13 & $\begin{array}{c}\text { Infección } \\
\text { urinaria }\end{array}$ & Coraliforme & Renal & NLP & No & Bien \\
\hline Varón & 2,5 & Dolor & 4 & $\begin{array}{c}\text { Ureteral } \\
\text { distal }\end{array}$ & URSC & No & Bien \\
\hline Varón & 4 & Dolor & 5 & $\begin{array}{c}\text { Ureteral } \\
\text { distal }\end{array}$ & URSC & $\mathrm{Si}$ & Bien \\
\hline Mujer & 3 & Dolor & 4,5 & $\begin{array}{c}\text { Ureteral } \\
\text { distal }\end{array}$ & URSC & No & Bien \\
\hline Mujer & 5,5 & Dolor & 6 & $\begin{array}{l}\text { Ureteral } \\
\text { proximal }\end{array}$ & URSC & No & Bien \\
\hline Varón & 8 & Dolor & 7 & $\begin{array}{c}\text { Ureteral } \\
\text { distal }\end{array}$ & URSC & $\mathrm{Si}$ & Bien \\
\hline Varón & 4 & Hematuria & 4 & $\begin{array}{c}\text { Ureteral } \\
\text { distal }\end{array}$ & URSC & $\mathrm{Si}$ & Bien \\
\hline
\end{tabular}

Con un tiempo de seguimiento que varía de 1 a 13 años todos los niños están asintomáticos, la ECO dentro de la normalidad y con buena función renal. No han presentado complicaciones ni a corto ni a largo plazo.

\section{DISCUSIÓN}

La incidencia de la nefrolitiasis oscila de 0-5\% tanto en Europa como en Estados Unidos ${ }^{1}$. Varía en función de la localización geográfica, raza, época el año y estatus socioeconómico ${ }^{2}$. Este último factor ha provocado un cambio en el hábito alimenticio, consistente en el aumento de la ingesta proteica, de carbohidratos refinados y de sodio $^{3}$. En un principio se pensó que esto podía repercutir solamente en la composición de las litiasis, pero no es así, también se ha demostrado su influencia en la localización de las mismas en el tracto urinario. En Europa en el inicio del siglo pasado, los cálculos más frecuentes en la edad pediátrica eran aquellos cuyo componente principal era el urato amónico y ubicados en la vejiga. Sin embargo en la actualidad han sido substituidos por los compuestos de oxalato cálcico y situados en el trayecto ureteral ${ }^{3}$. Millar et al en 1993 comunicaron que de su serie de 221 niños diagnosticados de litiasis, 202 la presentaban en el tracto urinario superior ${ }^{4}$. La litiasis vesical persiste como la más prevalente en países en vía de desarrollo y su aparición en nuestro medio se relaciona con alteraciones en este órgano como puedan ser las reconstrucciones y las disfunciones.

La urolitiasis en la edad pediátrica es un problema creciente ${ }^{2}$. Tarak Srivastava et al muestran esta evolución haciendo referencia a que Lattimer et al en 1951 en el Hospital Babies de Nueva York de 21.835 niños ninguno presentó patología litiásica ${ }^{5}$, y sin embargo Stapleton et al en 1987 comunicaron una serie de 112 niños con esta enfermedad 6 .

El escenario actual para la urolitiasis en la edad pediátrica es el de una patología en ascenso, recidivante y cuyo lugar de presentación es en el tracto urinario superior. Por lo cual se asemeja a su comportamiento en la edad adulta. Esto ha provocado que se intente trasladar la experiencia acumulada y las técnicas quirúrgicas ampliamente extendidas en esta edad al niño, con la intención de disminuir la invasividad y agresividad de los tratamientos consiguiendo altas tasas de resolución.

Omitiendo el tratamiento urgente de la litiasis urinaria, las opciones de que disponemos para esta patología son: observación, litotricia extracorpórea por ondas de choque (LEOC), ureterorrenoscopia (URSC), nefrolitotomía percutánea (NLP), cirugía laparoscópica, cirugía robótica y cirugia a cielo abierto. En ocasiones se utilizan varias conjuntamente. Al programar el trata- 
miento de la urolitiasis en el prepúber es esencial valorar la edad y talla del niño; tamaño, localización y composición del cálculo; alteraciones anatómicas de la vía urinaria y el material del que disponemos (podemos encontramos con desproporción entre el nefroscopio y el cáliz renal). Van Savage valoró la litiasis ureteral distal y otorga una gran importancia al tamaño del cálculo, asî relacionó el porcentaje de paso a vejiga de la litiasis en función del tamaño de éste y obtuvo que si era $<1 \mathrm{~mm}$ de diámetro pasaban el $58 \%$ espontáneamente a vejiga, si eran de $1-2 \mathrm{~mm}$ lo hacian un $65 \%$, los de $2-3 \mathrm{~mm}$ solamente un $33 \%$ y los $>4 \mathrm{~mm}$ no pasaban ninguno ${ }^{7}$.

La LEOC fue introducida como opción terapéutica en 1982 y revolucionó el tratamiento de esta patología ${ }^{8}$. Hoy en día se acepta tanto en niños como en adultos como la primera opción terapéutica pues está sobradamente demostrada su eficacia y seguridad ${ }^{9,10}$. Para demostrar que es un procedimiento seguro Goel M.C. et al realizaron prospectivamente un estudio en 50 niños entre 2 y 12 años sometidos a LEOC. Midieron antes y después de la misma los posibles cambios funcionales y morfológicos en la unidad renal. Para valorar la función midieron la tasa de filtración glomerular mediante la realización de un renograma con tecnecio-99 ácido radionucleótido (DTPA). Con un seguimiento de hasta 31 meses constataron que no se producían cambios. Las alteraciones morfológicas las evaluaron mediante la realización de ECO. Llegaron a objetivar 6 hematomas, todos se resolvieron espontáneamente sin requerir intervención ni dejar secuelas. Concluyeron que es un procedimiento seguro incluso en riñones en crecimiento ${ }^{11}$. Muslumanoglu et al para comprobar su efectividad publicaron su experiencia en 344 niños que presentaban litiasis en la pelvis renal. En función del tamaño del lito obtuvieron distintos porcentajes de éxito: con litiasis $<1 \mathrm{~cm}, 1-2 \mathrm{~cm}, \mathrm{y}>2 \mathrm{~cm}$ una tasa de éxito de $92 \%, 68 \%$ y $50 \%$ respectivamente, requirieron una media de 1,9 tratamientos ${ }^{12}$.

La NLP fue empleada para el tratamiento de la patología litiásica al final de la década de los 70 , pero hasta 1990 no se desarrolló el instrumental específico para la edad pediátrica ${ }^{14}$. Sin embargo la primera NLP en esta edad se realizó en $1985^{15}$. A la hora de desarrollar la técnica hay múlti- ples posibilidades, de este modo vemos como en muchos centros se coloca una nefrostomía sobre la unidad renal afecta el día previo a la intervención, para posteriormente en el quirófano tener ya el trayecto localizado ${ }^{14}$. Nosotros al igual que otros grupos ${ }^{16}$ hemos realizado el trayecto el día de la intervención con el fin de evitar dos procedimientos anestésicos al niño.

Para la punción del cáliz inferior lo hacemos bajo control fluoroscópico, solamente guiados por un criterio de mayor experiencia en esta técnica, hay grupos que defienden realizarlo bajo control ecográfico pues afirman obtener los mismos resultados y producir una menor irradiación del niño ${ }^{17}$.

Los resultados obtenidos dependen mucho de la experiencia del cirujano, del material de que se dispone y de la presencia de adversidades como anomalías anatómicas $\mathrm{u}$ otras, pero en general hay consenso en que se obtienen excelentes resultados que varian del $80-90 \%$ de enfermos libres de restos litiásicos ${ }^{16}$. En el único procedimiento que hemos realizado se consiguió la extracción de toda la masa litiásica, dejando la vía urinaria limpia. En algunas ocasiones en el caso de que les quede abundante masa litiásica se puede proceder posteriormente a repetir el procedimiento aprovechando que ya tienen el trayecto completado:"segundo vistazo" o bien probar con LEOC si los fragmentos residuales no son muy significativos.

La tasa de complicaciones con esta técnica quirúrgica es baja, la mayoría de los autores hacen referencia hasta un 5\% de complicaciones en sus casos. Las más frecuentemente descritas son: sepsis, sangrado, perforación de la vía y $\operatorname{otras}^{18}$. La mayoría de las ocasiones se relaciona directamente con la desproporción de tamaño entre el paciente y el instrumental ${ }^{17}$.

El hecho de dejar una nefrostomía de descarga o no es opcional. Es un tema en el que hay diversidad de opiniones, pero ningún estudio prospectivo realizado. Nosotros pese a ser un caso sin complicaciones decidimos dejarla para conseguir un mejor drenaje de la unidad renal y para tener el trayecto tutorizado por si se hacía necesario un "segundo vistazo" por quedar masa litiásica (aunque posteriormente no fue necesario). 
La NLP es un procedimiento seguro, Dwaba et al desarrollaron un estudio mediante la realización de un renograma con tecnecio -99 ácido dimercapto succínico (DMSA) y DTPA antes y después de la intervención en el que demostraron que no se producían cambios ${ }^{16}$.

Cuando la litiasis se presenta en localización ureteral, sobre todo en los tercios distal y medio el procedimiento de elección es la URSC. En general es un procedimiento sencillo y con excelentes resultados. La tasa de éxito varía de 75$100 \%$ dependiendo de la localización y tamaño del cálculo ${ }^{19}$. En una de las series más amplias Bassiri et al describen 66 procedimientos en 66 niños, el tamaño medio de la litiasis era de $8 \mathrm{~mm}$ y consiguieron hasta un $88 \%$ de éxitos ${ }^{20}$. En nuestra experiencia la tasa de éxito para este procedimiento aislado es menor (50\%), hemos tenido que reconvertir 3 de los enfermos a cirugía abierta. Creemos que se ha debido a la falta de material adecuado y de hábito en el manejo de este instrumental.

La dilatación del meato ureteral es un procedimiento opcional. Pretende aumentar el diámetro del meato ureteral de un modo controlado para evitar gestos más traumáticos posteriormente que pudieran lesionar esta unidad anatómica. Nosotros lo hemos realizado sistemáticamente en todos los niños a los que hemos realizado una URSC, no solo con fin de tratar la patología litiásica, sino también para resolver la estenosis de la unión pieloureteral y nunca hemos encontrado ninguna complicación intra ni posquirúrgica. Tras dicha maniobra los enfermos no desarrollan estenosis ni reflujo vésico-ureteral ${ }^{21}$.

Las complicaciones son muy infrecuentes, entre ellas se han descrito hematuria, perforación ureteral, infección y otras ${ }^{7}$, todas por lo general poco importantes y de fácil resolución. Bassiri et al en sus 66 procedimientos tuvieron $4,5 \%$ de pielonefritis y $16,6 \%$ de hematuria.

La colocación de un catéter ureteral tras el procedimiento es opcional. En nuestros casos lo hemos dejado siempre, pues entendemos que mantiene dilatada la vía facilitando la posterior eliminación de posibles restos. Además creemos que puede prevenir el desarrollo de complicaciones en el postoperatorio inmediato al mantener el meato cateterizado en el periodo en que se resuel- ve la inflamación y edema secundarios al trauma que supone esta técnica. En contra hay estudios que demuestran que la cateterización ureteral en la edad pediátrica produce mayor disconfort y se tolera peor que en los adultos ${ }^{14}$. Una postura razonable es la de no dejarlo de rutina y hacerlo únicamente en aquellos casos en que el procedimiento sea más traumático de lo habitual ${ }^{14}$.

Hay consenso de que con el desarrollo de nuevo material endourológico como ureterorrenoscopios semirrigidos y flexibles de menor tamaño, con canales de trabajo mayores; desarrollo del láser Holmium; nuevos dispositivos electrohidraúlicos y ecográficos para fragmentar la litiasis; material para extraer los fragmentos como las cestas de nitinol; y la experiencia del cirujano se conseguirán tasas de éxito muy cercanas al $100 \%$. No obstante siempre quedará un reducido número de casos que por su gran complejidad requerirán de la cirugía clásica a cielo abierto o vía laparoscópica ${ }^{22}$.

\section{CONCLUSIONES}

La experiencia acumulada a lo largo de los años ha demostrado que el abordaje endourológico en la edad pediátrica es factible y seguro. Hoy en día se debe convertir en la técnica de elección ante el fracaso de la LEOC. El desarrollo del nuevo material adaptado a esta edad y la experiencia del cirujano son las claves para el desarrollo definitivo de la técnica.

\section{REFERENCIAS}

1. Orson W. Kidney stones: pathophysiology and medical management. Lancet 2006;367(9507):333-344.

2. Srivastava T., Alon US. Urolithiasis in adolescent children. Adolesc Med Clin 2005;16(1);87-109

3. Trincheri A. Epidemiology of urolithiasis. Arch Ital Urol Androl 1996;68(4):203-249.

4. Milliner DS, Murphy ME. Urolithiasis in pediatric patients. Mayo Clin Proc 1993;68(3):241-248.

5. Lattimer JK, Hubbard M. Pediatric urologic admissions. J Urol 1951; 66:289-293.

6. Stapleton FB, Mckay CP, Noe HN. Urolithiasis in children: the role of hypercalciuria.. Pediatr Ann 1987;16(12):9809811, 984-992.

7. Van Savage JG, Palanca LG, Andersen RD, Rao GS, Slaughenhoupt BL Treatment of distal ureteral stones in children: similarities to the american urological association guidelines in adults J Urol 2000 Sept; 164(3pt2): 1089-1093.

8. Chaussy C, Schmiedt E, Jocham D, Brendel W, Forssmann B, Walther V. First clinical experience with extracorporeally induced destruction of kidney stones by shock waves. J Urol 1982;127(3):417-420. 
9. Shukla AR, Hoover DR, Homsy YL, Perlman S, Schurman $\mathrm{S}$, Reisman EM. Urolithiasis in the low birth weigth infant: the role and efficacy of extracorporeal shock wave lithotripsy. J Urol 2001 Jun;165(6 pt 2):2320-2323.

10. Lottmann Hb, Archambaud F, Traxer O, Mercier-Pageyral B, Helal B. The efficacy and parenchymal consequences of extracorporeal shock wave lithotripsy in infants. BJU Int. 2000 Feb;85(3):311-315.

11. Goel MC, Baserge NS, Babu RV, Sinha S, Kapoor R. Pediatric kidney: functional outcome after extracorporeal shock wave lithotripsy. J Urol. 1996 Jun;155(6):20442046.

12. Muslumanoglu AY, Tefekli A, Sarilar O, Binbay M, Altunrende F, Ozkuvanci U. Extracorporeal shock wave lithotripsy as first line treatment alternative for urinary tract stones in children: a large scale retrospective analysis. J Urol. 2003 Dec;170(6 Pt 1):2405-2408.

13. Esen T, Krautschick A, Alken P. Treatment update on pediatric urolithiasis. World J Urol. 1997;15(3):195-202. Review.

14. Durkee CT, Balcom A. Surgical management of urolithiasis. Pediatr Clin North Am. 2006 Jun;53(3):465-477, vii. Review.

15. Woodside JR, Stevens GF, Stark GL, Borden TA, Ball WS. Percutaneous stone removal in children. J Urol. 1985 Dec; 134(6): 1166-1167.

16. Dawaba Ms, Shokeir AA, Hafez AT, Shoma AM, ElSherbiny MT, Mokhtar A et al. Percutaneous nephrolithotomy in children: early and late anatomical and functional results. J Urol. 2004 Sep;172(3):1078-1081
17. Desai M, Ridhorkar V, Patel S, Bapat S, Desai M. Pediatric percutaneous nephrolithotomy: assessing impact of technical innovations on safety and efficacy. J Endourol. 1999 Jun; 13(5):359-364

18. Minevich E, Defoor W, Reddy P, Nishinaka K, Wacksman J, Sheldon $\mathrm{C}$ et al. Ureteroscopy is safe and effective in prepubertal children. J Urol. 2005 Jul;174(1):276-279; discussion 279.

19. Desai M.. Endoscopic management of stones in children. Curr Opin Urol. 2005 Mar;15(2):107-112. Review.

20. Bassir A, Ahmadnia H, Darabi MR, Yonessi M. Transureteral lithotripsy in pediatric practice. J Endourol. 2002 May; 16(4):257-260.

21. Jayanti VR, Arnold PM, Koff SA Strategies for managing upper tract calculi in young children. J Urol. 1999 Sep;162 (3 Pt 2):1234-1237.

22. Alivizatos G., Skolarikos A. Is there still a role for open surgery in the management of renal stones? Curr Opin Urol. 2006 Mar;16(2):106-11. Review.

Correspondencia autor: Dr. J. Romero Otero Servicio de Urología. Hospital 12 de Octubre. Avda. de Córdoba, s/n - 28041 Madrid

Tel.: 913908121

E-mail autor: jromerootero@hotmail.com

Información artículo: Original - Urología pediátrica

Trabajo recibido: diciembre 2006

Trabajo aceptado: febrero 2007

\section{COMENTARIO EDITORIAL}

Reconociendo que el artículo es metodológicamente correcto y apto para su publicación, no quisiéramos que mediante el mismo se pudiera transmitir el concepto erróneo de que cualquier calcúlo en la vía urinaria en niños debe ser tratado mediante manejo endourológico, sino que por el contrario deben ajustarse extremadamente sus indicaciones, e igualmente que estas técnicas no están exentas de potenciales y graves riesgos y por ello a nuestro parecer, deben realizarse por manos muy expertas y con los medios adecuados. Así pues, le agradecería trasmitiera las siguientes cuestiones y comentarios a sus autores:

1) Nos gustaría conocer ¿cuáles son las indicaciones del autor para realizar la ureterorrenoscopia en el manejo de la litiasis en niños?, pues llama la atención que solo dos de los cálculos superan el tamaño aconsejado para aplicar cualquier tipo de tratamiento $(5 \mathrm{~mm})^{1}$, máxime cuando casi todos se encontraban ya en uréter distal y como es sabido los niños expulsan con facilidad estos cálculos. Y en caso de que la indicación fuese por persistencia del dolor u obstrucción si ¿no podrían haberse resuelto mediante la simple colocación de un doble-J sin añadir el riesgo de la dilatación meatal y de la manipulación uretrorrenoscópica?.

2) Nos gustaría conocer ¿qué flujos y presiones de instilación del líquido de irrigación emplea para realizar la ureterorenoscopia?, pues se trata de un dato importante dado que elevadas presiones incrementan el riesgo de perforación o por el contrario, flujos insuficientes predisponen también a la perforación por manipulación con visualización deficiente.

3) Reconocemos la habilidad del autor para realizar ureterorrenoscopia empleando un cistoscopio 9`5 F, hecho que verdaderamente es posible, e igualmente desconocemos el lugar o país de trabajo de los autores, pero no es menos cierto que las técnicas de ureterorrenoscopia no están exentas de posible yatrogenia $(9-11 \%)^{2,3}$, siendo incluso algunas muy graves como la perforación y la avulsión ureteral. Asimismo, es un hecho demostrado que existe una fuerte relación entre la tasa de complicaciones y el instrumental 
empleado y la experiencia del urólogo ${ }^{3}$. Por ello y considerando que existen instrumentos de diseño y calibre específicos $\left(6-7^{`} 5 \mathrm{~F}\right)$ para la población infantil $^{3}$ que evitan realizar la dilatación del meato en casos donde sería imprescindible para un 9`5 F, con bombas de instilación de flujo adecuados y que se utilizan habitualmente por manos expertas en algunos de los Servicios de Urología Infantil de nuestro país, nos gustaría saber ¿cómo justifica su realización? o si por el contrarío ¿no sería más correcto remitirlos a centros con el utillaje y la experiencia adecuados a su patología y edad?

1. S. Tekgül, H. Riedmiller, D. Beurton, E. Gerharz, P. Hoebeke,R. Kocvara, Chr. Radmayr, D. Rohrmann. http:// www. uroweb.org/fileadmin/user_upload/Guidelines/19\%20Pae diatric\%20Urology.pdf

2. Johnson DB, Pearle MS. Complications of ureteroscopy. Urol Clin North Am. 2004;31(1):157-171

3. H.-G. Tiselius, D. Ackermann, P. Alken, C. Buck, P. Conort, M. Gallucci, T. Knoll. http://www.uroweb.org/fileadmin/ user_upload/Guidelines/18\%20Urolithiasis.pdf.

Fdo.: Dr. Agustin Serrano

\section{REPLICA AL COMENTARIO EDITORIAL}

En ningún momento este artículo transmite el "concepto erróneo de que cualquier cálculo deba ser tratado endourológicamente”, sino más bien todo lo contrario, que es una técnica que se reserva para el fracaso de la actitud expectante o litotricia extracorpórea, de hecho todos nuestros casos como se refleja en el escrito, fueron previamente tratados con litotricia.

Como autores del artículo quisiéramos responder a los comentarios expuestos por el Dr. Agustín Serrano, precisando las siguientes puntualizaciones:

1) El artículo expone que el tratamiento endourológico no es una indicación primaria para tratar las litiasis del trato urinario superior (TUS), sino una opción terapéutica muy buena, incluso hay autores que la consideran como el "gold estándar"1, cuando otros tratamientos más corrientes, conservador y litotricia extracorpórea, han fracasado. Además pensamos que tenemos la suficiente experiencia para efectuar este tipo de técnicas que no se practican en todos los centros, siendo el motivo de su publicación.
En nuestra serie la indicación de la extracción del cálculo se ha hecho en base a la persistencia de cuadro clínico, dolor tipo cólico, con obstrucción de la vía urinaria y falta de eliminación de la litiasis tras tratamiento conservador, en un tiempo prudencial, o intento de litotricia, si la situación lo ha permitido. Creemos que no todos los cálculos menores de $5 \mathrm{~mm}$ en el uréter se eliminan con facilidad. El tamaño del cálculo es un factor crucial para la expulsión del mismo, pero en primer lugar hay excepciones y en segundo lugar el tamaño más extendido en la literatura científica para la edad pediátrica es el de $4 \mathrm{~mm}$, como se refleja en la discusión de nuestro escrito (referencia 7 del mismo). Hay múltiples publicaciones que tratan los de $3 \mathrm{~mm}^{2-7}$, basándose en la situación del paciente. En nuestros casos, los menores tenían $4 \mathrm{~mm}$ y la situación clínica de los niños aconsejaba su intento de extracción.

En cuanto a la utilización del catéter doble J para solventar la situación clínica, hay que puntualizar que en la infancia requiere de anestesia general, cistoscopia y es, a veces, difícil su correcta situación, por lo que el intento de extracción de la litiasis y su resolución no aumenta de forma ostensible la morbilidad del paciente. Además, una vez colocado puede interrumpir la salida del cálculo, ya que provoca una pequeña dilatación del meato de forma pasiva, y sólo alivia temporalmente el cuadro obstructivo. Nosotros lo ponemos temporalmente cuando se ha fragmentado la litiasis o se ha manipulado dentro del uréter, evitando obstrucciones secundarias por estos motivos. Preferimos dilatar el meato ureteral en la mayoría de los casos, pues no aumenta el riesgo y sí permite el paso de catéteres, cistoscopioureterenoscopio y la extracción del cálculo de forma mucho más segura (la dilatación del meato se practica en otras unidades o secciones de nuestro país por diversas patologías sin referir un aumento de la morbilidad, encontrando diferentes autores que también lo realizan ${ }^{1-7}$. Nosotros también la practicamos asociada a otros procedimientos, como paso de sonda Acucise, paso de ureterenoscopios, etc., sin complicaciones.

2) No utilizamos un flujo especial, sino el que permite el canal del ureterorenoscopio o cistoscopio del 7,5 y 9,5 Fr., colocando la bolsa de infu- 
sión a una altura aproximada de $1 \mathrm{~m}$ por encima del paciente, sin utilizar nunca bomba de perfusión. Sólo cuando hay dificultad de visualización, la compresión suave de la bolsa de perfusión por el ayudante permita dominar la situación, siempre bajo visión directa. Con esta metodología no se han producido perforaciones ureterales.

3) Tenemos un ureterenoscopio rígido del 7,5 Fr. que se utiliza para alcanzar el uréter medio y el proximal, aunque como refiere el artículo, fracasamos en la extracción del cálculo en el uréter proximal. En los casos de acceso al uréter distal preferimos emplear, si la dilatación nos los permite, el cistoscopio telescopado del 9`5 Fr. con canal recto (descrito hace años por Young ${ }^{7}$ ), ya que tiene un buen de canal de trabajo y es de fácil maniobrabilidad ${ }^{7}$ (el manejo del ureterorenoscopio en la infancia es más difícil en el uréter distal por su longitud). Además, éste sólo se introduce uno pocos $\mathrm{cm}$, normalmente $2-3 \mathrm{~cm}$, desde la unión, permitiendo visualizar la litiasis. Como hemos comentado no creemos que la dilatación represente un peligro, sino más bien un paso necesario para evitar complicaciones producidas por el paso del cistoscopio - ureterorenoscopio en la unión urétero-vesical, permitiendo posteriormente, de forma más fácil, la salida del cálculo sin desgarros.

Estamos de acuerdo que este procedimiento se debe efectuar en Secciones o Unidades de Urología Infantil con experiencia (Servicios que en nuestro país aún no existen), por manos expertas en el manejo endoscópico y con material adecuado que ha sido la causa por lo que noso- tros lo hemos aplicado, ya que consideramos que tenemos la suficiente experiencia en la realización de dichas técnicas endourológicas, siendo nuestra Sección una de las que son punto de referencia para dichos procedimientos en nuestro país.

Para finalizar quisiéramos añadir unas citas bibliográficas más, además de las referidas en el artículo, donde se puede leer muchos de los razonamientos expuestos en este comentario.

\section{REFERENCIAS}

1. Ureteroscopical treatment of ureterolithiasis in childhood. Our experience. Touloupidis S, Papathanasiou A, Fotas A, Salpiggidis G, Haralambous S, Rombis V. Arch Ital Urol Androl. 2006 Mar;78(1):20-22.

2. Safety and outcome of rigid ureteroscopy for management of ureteral calculi in children. El-Assmy A, Hafez AT, Eraky I, El-Nahas AR, El-Kappany HA. J Endourol. 2006 Apr;20(4): 252-255.

3. Pediatric ureteroscopic stone management. Thomas JC, DeMarco RT, Donohoe JM, Adams MC, Brock JW 3rd, Pope JC 4th. J Urol. 2005 Sep; 174(3):1072-1074.

4. Ureteral access sheath for the management of pediatric renal and ureteral stones: a single center experience. Singh A, Shah G, Young J, Sheridan M, Haas G, Upadhyay J. J Urol. 2006 Mar;175(3 Pt 1):1080-1082; discussion 1082.

5. Rigid ureteroscopy for the treatment of ureteral calculi in children. Satar N, Zeren S, Bayazit Y, Aridogan IA, Soyupak B, Tansug Z. J Urol. 2004 Jul;172(1):298-300.

6. Complications of 2735 retrograde semirigid ureteroscopy procedures: a single-center experience.Geavlete P, Georgescu D, Nita G, Mirciulescu V, Cauni V. J Endourol. 2006 Mar;20(3):179-185.

7. Endourology for stone disease. A. Shulkla and M. Eihard. In The Kelalis- King- Belman Textbook of Clinical Pediatric Urology. Fifth edition. Steven G. Docimo. Informa Healthcare. 2007. Cap 26; 401-420.

Fdo.: Romero Otero J et al. 